Введение. Оказание медицинской помощи пациентам с повреждением и несостоятельностью швов пищевода остается обсуждаемой и актуальной темой.

Цель исследования - представить обзор литературы с результатами данных по диагностике и методам лечения перфораций пищевода (ПП), обратив особое внимание на группу пациентов с поздними сроками обращения, имеющих разного рода осложнения.

Материал и методы. Проанализированы опубликованные отечественными и зарубежными авторами данные и представлены результаты лечения пациентов с ПП в отделении торакальной хирургии УЗ «Брестская областная клиническая больница».

Результаты. Отражены литературные данные по лечению ПП с применением разных методик и представлены результаты лечения пациентов в отделении торакальной хирургии УЗ «Брестская областная клиническая больница» с применением оригинальной методики с установкой T-образной разборной дренажной системы с формированием «контролируемой фистулы». Летальность пациентов при эндоскопических вмешательствах при ПП составила 10\%.

Выводы. При ПП, по данным разных авторов, общая летальность на сегодняшний день достигает $67 \%$. Использование видеоторакоскопических вмешательств при ПП позволяет снизить летальность до $10 \%$.

Ключевые слова: перфорация пищевода, несостоятельность швов, видеоторакоскопия

\title{
ESOPHAGEAL PERFORATIONS: DIAGNOSIS AND TREATMENT
}

A. N. Ignatsiuk, A. S. Karpitski

Brest Regional Clinical Hospital, Brest, Belarus

Background. Rendering medical care to patients with damage and failure of esophageal sutures remains a controversial and relevant topic.

Objective. To provide a literature review on the diagnosis and treatment of esophageal perforation (EP), paying particular attention to the group of patients with delayed admission to the hospital and with various complications.

Material and methods. The data published by domestic and foreign authors are analyzed and treatment outcomes of patients with EP admitted to the Department of thoracic surgery of Brest Regional Clinical Hospital are presented.

Results. The literature data on EP treatment options are reflected and clinical outcomes of EP patients treated in the Department of thoracic surgery of the Healthcare Institution 'Brest Regional Clinical Hospital' using an original technique involving installation of a collapsible T-shaped drainage system with the formation of a "controlled fistula" are presented. The mortality rate of patients with endoscopic interventions for PN amounted to $10 \%$.

Conclusions. Currently, according to various authors, the overall EP mortality rate reaches $67 \%$. The use of videothoracoscopic interventions for EP can reduce the mortality up to $10 \%$.

Keywords: esophageal perforation, suture failure, videothoracoscopy

Автор, ответственный за переписку:

Игнатюк Александр Николаевич, УЗ «Брестская областная клиническая больница»;

e-mail: alexihnatsiuk@gmail.com

Для цитирования: Игнатюк, А. Н. Перфорации пищевода: диагностика и лечение / А. Н. Игнатюк, А. С. Карпицкий // Гепатология и гастроэнтерология. 2021. Т. 5, № 1. С. 37-43. https://doi.org/10.25298/2616-5546-2021-5-1-37-43

\section{Corresponding author:}

Ignatyuk Aleksandr Nikolaevich; Health care institution «Brest Regional Clinical Hospital» e-mail: alexihnatsiuk@gmail.com

For citation: Ignatsiuk AN, Karpitski AS. Esophageal perforations: diagnosis and treatment. Hepatology and Gastroenterology. 2021;5(1):37-43. https://doi. org/10.25298/2616-5546-2021-5-1-37-43
Несмотря на стремительное развитие современных медицинских технологий, оказание помощи пациентам с повреждением и несостоятельностью швов пищевода остается обсуждаемой и актуальной темой. Этиологию фракторов перфораций пищевода (ПП) можно разделить на три большие группы: ятрогенные, барогенные (спонтанные) и травматические.

Роль эндоскопических методов в диагностике болезней пищевода возрастает и принимает статус скринингового метода, что неизбежно увеличивает количество осложнений - ПП, доля которых составляет от 60 до 80\% всех повреждений [1]. По данным А. А. Шалимова (1975) и S. Richard (2002), при лечебной эндоскопии процент повреждений пищевода возрастает от 2,6 до 4\%. Особо актуальна категория пациентов с ахалазией кардии, где при выполнении баллонной дилатации ПП отмечается в 1,4-4,9\% случаев, у некоторых исследователей - до 20\% $[2,3]$. При бужировании постожоговых стриктур пищевода разрывы выявляются в 1-10,3\% случаев [4]. Смертность от ПП варьирует от 3 до $67 \%$ и в среднем на современном этапе состав- 
ляет 19,7\% [5]. Следует особо отметить, что по результатам проводимого нами лечения количество летальных исходов от ПП составило 11\% (7 пациентов).

На долю спонтанных разрывов пищевода приходится от 10 до 35\% всех перфораций [6]. В отделении торакальной хирургии УЗ «Брестская областная клиническая больница» (УЗ «БОКБ») доля спонтанных разрывов от всех перфораций - 31\% (19 пациентов). В отличие от инструментального воздействия и повреждения инородным телом, когда известен этиологический фактор, диагностика спонтанного разрыва может представлять большие сложности, операция нередко выполняется при развитии осложнений - пиопневмоторакса, эмпиемы плевры, гнойного медиастинита, что и приводит к неблагоприятным результатам. Послеоперационная летальность варьирует от 25 до 85\% и находится в прямой зависимости от времени, прошедшего с момента ПП [7]. Лучшие результаты связаны с оптимальным сроком лечения, ранней диагностикой и правильно выбранным оперативным вмешательством в течение 12 часов. Оказание хирургической помощи позднее 24 часов после ПП повышает риск летального исхода до $50 \%$ и более [8].

В медицинской литературе постоянно публикуются материалы о разнообразии инородных тел и отмечается, что количество повреждений пищевода составляет 30-77\% [9]. Летальность при инородных телах пищевода не превышает $1 \%$, но при развитии осложнений (медиастинит, эмпиема) возрастает до 40-45\% [10]. Следует отметить, что 17 случаев (28\%) ПП у пациентов, пролеченных в нашем стационаре без летальных исходов, были представлены повреждениями, наступившими при извлечении инородного тела.

В зависимости от локализации ПП в разных его отделах определяется полиморфность клинических проявлений и, как следствие, - хирургических подходов к лечению данных пациентов. ПП в грудном отделе в большинстве случаев проявляется как острое заболевание.

Контрастное рентгеноскопическое исследование пищевода остается доказательным и широко применяемым методом диагностики повреждений пищевода, чувствительность которого составляет 73-95\% [11]. Для выявления ПП данный метод диагностики нами применен в $48 \%$ случаев.

Осторожное применение эзофагоскопии при ПП обусловлено, во-первых, риском нарастания пневмоторакса, вплоть до напряженного, во-вторых, опасностью возникновения и нарастания подкожной эмфиземы и эмфиземы средостения, что создает недостоверную картину повреждения пищевода и подталкивает хирурга к необоснованному хирургическому вмешательству.
Чувствительность и специфичность данной методики - 100\% и 83-92,4\%, соответственно [12]. Дополнением для установления локализации ПП служит применение эзофагоскопии во время оперативного вмешательства.

Спиральная компьютерная томография (СКТ). Исследование имеет ряд преимуществ перед традиционными рентгенографическими методиками, обладает чувствительностью $100 \%$, специфичностью - 96\% [13]. В комбинации с приемом водорастворимого контрастного препарата с успехом выявляются затеки за стенку пищевода. Высокая чувствительность методики позволяет дифференцировать отдельные органы и ткани по плотности в пределах 0,5\%. При отсутствии повреждения медиастинальной плевры появляется возможность выявить скопление газа в клетчатке средостения, его расширение, признаки отека, размозжения и утолщения пищеводной стенки. СКТ с контрастированием пищевода нами выполнена в $52 \%$ случаев ПП, причем надо отметить, что в 19\% случаев данный метод дополнил рентгеноскопию ввиду сомнительных диагностических данных.

Существует два вектора в направлении лечения повреждений пищевода: консервативный и хирургический. По утверждению K. L. Franco et al. (1998), выбор метода определяют следующие факторы: этиология, распространенность повреждения и длительность заболевания; наличие изменений со стороны пищевода; состояние пациента и его возраст [14].

ПП в несколько миллиметров без признаков сепсиса и преимущественно ятрогенной этиологии уместно лечить консервативными методами. Под консервативным лечением понимают исключение перорального питания не менее 1-2 недель, гигиена полости рта, полноценное сбалансированное парентеральное или энтеральное питание, применение широкого спектра антибиотиков, размещение эзофагеального отсасывающего дренажа [15]. Применение консервативной терапии возможно при небольших ограниченных средостением дефектах пищевода, выявленных в первые 24-48 часов, при которых рентгеноконтрастное вещество не распространяется за пределы стенки более чем на 1,5-2 см. При этом образовавшееся депо хорошо опорожняется в просвет пищевода, симптомы перфорации и признаки сепсиса минимально выражены. Правильная оценка первичного состояния пациентов при ПП способствует снижению летальности в группе консервативного лечения с 20-38\% до 0\% [16]. Примерно у $20 \%$ пациентов данной категории, даже при строгом соблюдении всех вышеперечисленных критериев, может потребоваться срочное хирургическое вмешательство. Когда же консервативные подходы оказывались безуспешными и требовалось хирургическое вмешательство, количество 
осложнений и продолжительность нахождения в стационаре возрастали [17].

Как разновидность консервативного лечения, G. Santos и R. Frater (1986) предложили систему «чреспищеводного орошения средостения» пациентам с ПП, не диагностированной вовремя. Методика заключалась в установке зонда с множеством перфорационных отверстий на проксимальном конце к месту разрыва, а дренажную трубку размещали через плевральную полость к непосредственной близости пищевода. Через зонд производилось введение антисептиков, аспирацию осуществляли через зонд, тем самым обеспечивая орошение средостения [18].

I. C. Wesdorp et al. при ПП использовали назопищеводную аспирацию, помещая дренажную трубку в просвет пищевода таким образом, что дренажные отверстия располагались выше и ниже дефекта стенки. Аспирация проводилась 5-7 дней, по истечении которых наблюдалось закрытие дефекта. Летальность в данной группе составила 8\% [4].

Некоторые авторы относят к консервативному лечению эндоскопические хирургические вмешательства [19].

Метод эндоскопической вакуумной терапии G. Loske впервые применил для лечения спонтанного разрыва пищевода. Методика заключается в создании локального отрицательного давления через специальную пористую губку в месте перфорации, что способствует санации раневого секрета, притоку крови, фрормированию грануляций, заживлению раны [20].

R. Mennigen et al. в 2014 г. провели курс вакуумной эндоскопической терапии у 101 пациента с успешными результатами в 90\%. ПВХ-дренаж с синтетической губкой на проксимальном конце проводят через нос в просвет пищевода и устанавливают в свищевое отверстие. Создают разряжение 100-125 мм рт. ст., проводят в течение нескольких дней аспирацию до очистки раны и формирования грануляции [21].

В последнее десятилетие значительно увеличилось количество высокотехнологических внутрипросветных эндоскопических методов: закрытие раны пищевода эндоскопическими клипсами и over-the-scope clips (OTSC), установка саморасширяющихся пищеводных стентов, герметизация малых свищевых сообщений и укрепление швов пищевода цианоакриловым клеем $[5,21]$.

Стентирование применятся в лечении ПП, пищеводных свищей, несостоятельности пищеводно-желудочных и пищеводно-энтеральных анастомозов как основной метод, так и в качестве дополнения в этапном лечении [22-24]. После установки стента в просвет пищевода происходит восстановление непрерывности пищеводной трубки, устраняется попадание содержимого в плевральную полость, появляется возможность питания через рот, уменьшается число инфекционных осложнений [25]. Согласно литературным данным, эфффективность этого метода достигает 90\% при условии адекватного дренирования плевральной полости [26]. В 2006 г. A. Fischer сообщил об эндоскопической установке саморасширяющихся металлических стентов у 15 пациентов. R. Freeman et al. (2007) сообщили об установке стентов при повреждении пищевода. В результате успешное излечение произошло у 16 из 17 пациентов. Условием к успешному выздоровлению, подчеркивают авторы, является раннее введение стента после установления диагноза для исключения инфицирования средостения [27]. Основное из осложнений при установке стента - его миграция, что требует эндоскопической коррекции или замены [28]. Перфорация, наступившая в результате распада опухоли, либо инструментальная перфорация у онкологических пациентов - серьезное осложнение. Этой группе пациентов предпочтительнее установка покрытых саморасправляющихся стентов, особенно в случае образования свищей между пищеводом и дыхательными путями [10].

Превалирующая часть хирургического сообщества предпочитают только оперативный подход в лечении ПП. Впервые успешный оперативный опыт в лечении ПП применил J. Collins (1944), выполнив ушивание. N. Barret (1946) осуществил первое успешное ушивание при синдроме Бурхаве. A. Olsen et al. (1947), T. Kinsella (1948) также успешно пролечили разрыв пищевода, указав на отсутствие осложнений, связанных с непосредственным оперативным вмешательством. Таким образом, спонтанные разрывы пищевода, имея более высокий риск инфицирования средостения и плевральной полости, обоснованно подвергаются оперативному лечению [29].

Применяя активную хирургическую тактику при разрывах внутригрудного отдела пищевода, некоторые авторы рекомендуют ушивать ПП вне зависимости от сроков и состояния окружающих тканей [30-34]. Часть исследователей рекомендуют ограничиться дренированием плевральной полости, наложением эзофрагостомы с гастростомой либо произвести резекцию поврежденного участка пищевода [35].

Тяжелое состояние пациента в случае поздней диагностики вынуждает хирургов ограничиться применением только дренирования. Недостаток такого лечения - длительность (до 2-7 месяцев) стационарного лечения, что сопряжено с высокой летальностью. R. G. Berrisford et al. (2007), применяя дренирование области ПП, предложили дополнять лечение установкой ПВХ-трубки через просвет желудка. Таким образом создается отрицательное давление с двух сторон от стенки пищевода, исключается заброс содержимого желудка и пищевода в средосте- 
ние. Авторам удалось сократить пребывание пациентов в стационаре и улучшить отдаленные результаты. В нашей клинике одному пациенту ввиду тяжести состояния из-за сопутствующей патологии было выполнено только дренирование плевральной полости, пациент умер.

Место хирургического доступа зависит от уровня повреждения пищевода [36]. При ПП в шейном отделе предпочтителен доступ по передней поверхности кивательной мышцы, в основном слева. Данный доступ нами применен в 46,1\% случаев (6 пациентов), в остальных же случаях применена двусторонняя цервикотомия по передней поверхности кивательной мышцы со вскрытием клетчаточных пространств шеи ввиду быстро развивающейся фрлегмоны шеи. При поражении верхней и средней трети грудного отдела пищевода основной доступ - правосторонняя торакотомия, в нижней трети - левосторонняя торакотомия [37].

Тактика хирургического лечения в зависимости от срока разрыва пищеводной трубки на сегодняшний день четко не определена. При неопухолевых перфорациях, отсутствии некроза и постановке диагноза в течение 24 часов многие авторы считают методом выбора первичное наложение швов [12, 32, 34, 38]. P. N. Symbas et al. (1978) рекомендуют ушивание пищевода проводить в первые 12 часов. P. Linden (2007) при выполнении оперативного вмешательства (менее суток) сообщил о послеоперационной летальности в 5\%. Keeling et al. (2010) сообщили о летальности менее 10\% при первичном ушивании позднее 24 часов после повреждения. Основное осложнение, как правило, - несостоятельность швов пищевода. С целью ее предотвращения выполняется ряд вспомогательных методик увеличения прочности зоны ушивания при использовании ксеноперикарда, дна желудка, участка диафрагмы, межреберных мышц, плевры, пряди большого сальника [33, 39]. Однако ни одна из вышеперечисленных методик не гарантирует снижение летальности и не всегда приводит к уменьшению частоты несостоятельности [37] Так, Т. Н. Gouge сообщил, что при простом ушивании дефекта пищевода несостоятельность швов возникает в 39\% случаев, а при укрепленном - уменьшается до 14\%, смертность составляет, соответственно, 25 и 6\% [40].

Образование пищеводных свищей происходит в 10-38\% случаев после ушивания пищевода. К определяющим факторам развития осложнений относят небрежную и неадекватную хирургическую обработку раны, деваскуляризацию пищевода, натяжение по линии швов и инфекцию. Vogel et al. (2005) считают, что пищеводно-кожные свищи, формируемые методом дренажной трубки, излечимы, но такой оптимизм разделяют не все хирурги.
Лечение перфораций пищевода методом «выключения» пищевода впервые описано в 1974 г. Под функциональным «выключением» подразумевают сохранение анатомической непрерывности пищевода и возможность естественного пассажа. Производится установка назогастрального зонда и наложение гастро- или еюностомы. Анатомическое выключение сопровождается эзофагостомией, прошиванием или лигированием пищевода без возможности естественного пассажа. Монополярное «выключение» - это «выключение» выше или ниже участка перфорации пищевода, биполярное «выключение» - «выключение» выше и ниже участка перфорации [41].

По мнению некоторых хирургов, целесообразно временно «выключать» пищевод с целью предупреждения и профилактики несостоятельности швов [6]. Применяемый как самостоятельный, указанный метод лечения в последующем требует повторных оперативных вмешательств по восстановлению непрерывности желудочно-кишечного тракта ввиду плохого качества жизни пациентов [37].

O. A. Abbott (1970) предложил создавать «управляемую фристулу» в качестве альтернативы операции «выключения» пищевода. Сущность метода в установке Т-образной трубки в разрыв пищевода и выведение дистальной ее части на грудную стенку [42]. При этом происходит предотвращение попадания инфицированного содержимого в околопищеводную клетчатку и средостение, создается контролируемый пищеводно-плевро-кожный свищ, заживающий консервативно после удаления Т-образного дренажа [43]. Недостаток данного метода заключается в необходимости наложения гастростомы [33]. P. Linden (2007) устанавливал Т-образный дренаж при запоздалой диагностике перфорации, что позволило снизить смертность до 8,7\% [44]. Летальность в группе поздно выявленных перфораций с применением в лечении Т-образного дренажа сопоставима с таковой у пациентов, оперируемых в первые 24 часа [44].

В случае разрыва пищевода или тяжелого медиастинита, когда невозможно выполнить ушивание, методом выбора хирургического вмешательства становится экстирпация пищевода с одномоментной или отложенной реконструкцией $[38,45]$. Производить экстирпацию при тяжелой сопутствующей патологии и в пожилом возрасте, даже с пораженным рубцовым процессом пищевода, не следует [46].

На базе УЗ «БОКБ» хирургическое вмешательство в виде торакотомии выполнено в 29,5\% случаев (18 пациентам), поступившим с перфорацией грудного отдела пищевода. Из них экстирпация (в некоторых случаях резекция) пищевода выполнена в 33,4\% случаев; ушивание произведено в 16,6\%; дренирующие операции 
- в 27,7\%; установка Т-образной разборной дренажной системы - в 23,3\% случаев. Летальность при открытых вмешательствах при перфорациях пищевода составила 16,6\% (3 пациента).

Последние 20 лет стали появляться сообщения об использовании видеоторакоскопического и лапароскопического методов в лечении перфорации пищевода. Базисными целями видеоторакоскопии стали визуализация перфорации, очистка раны от некротических тканей, ушивание стенки пищевода, дренирование средостения и плевральной полости [47]. Ушивание стенки пищевода при видеоторакоскопии выполнялось как в первые часы, так и через сутки после перфорации $[48,49]$. H. J. Scott с соавт. (1995) использовали интракорпоральный шов для ушивания раны пищевода во время видеоторакоскопии [50]. Использование видеоторакоскопического метода дает возможность не только ушить разрыв пищевода, но и позволяет эффективно санировать и дренировать средостение и плевральную полость [49]. S. Radhika et al. (2000) полагают, что применение видеоторакоскопических методик оправдано в случае высокого риска оперативного вмешательства у пациентов с поздно диагностированными перфорациями. M. Chung et al. (2001) считают, что эндовидеохирургия показана пациентам с тяжелым состоянием для выполнения радикальной операции.

На базе УЗ «БОКБ» видеоторакоскопические (ВТС) вмешательства пациентам с ПП выполнены в 47,5\% случаев (29 пациентов). Из них 21\% (6 пациентов) - видеоторакоскопия с экстирпа- цией пищевода и одномоментной пластикой пищевода, 34\% (10 пациентов) - ВТС с фиксацией дренажа в предполагаемом месте перфорации стенки пищевода, 45\% (13 пациентов) - ВТС с установкой разборной Т-образной дренажной системы (патент № a 20130660 26.04.2016). Летальность пациентов при эндоскопических вмешательствах при ПП составила 10\%.

\section{Выводы}

Проблема оказания помощи пациентам с ПП продолжает оставаться в центре внимания хирургов и сегодня. Сложным видятся вопросы выбора метода лечения, оптимального операционного доступа, рационального объема вмешательства и методов послеоперационного ведения пациента. Имеется множество положительных моментов и преимуществ в каждом перечисленном методе, однако не существует идеального и универсального хирургического лечения при всех случаях перфорации. Пациентам, имеющим гнойно-некротические осложнения в месте ПП, не всегда можно выполнить первичное ушивание перфорации или реконструктивное одномоментное вмешательство ввиду высокого риска несостоятельности швов. В таких сложных случаях оптимальный метод - установка Т-образной разборной дренажной системы с формированием «контролируемой фистулы». Оперативные вмешательства необходимо выполнять с использованием минимально инвазивных технологий.

\section{References}

1. Scheinin SA, Wells PR. Esophageal Perforation in a Sword Swallower. Texas Heart Institute Journal. 2001;28(1):6568.

2. Ferguson MK. Achalasia: Current evaluation and therapy The Annals of Thoracic Surgery. 1991;52(2):336-342. doi: 10.1016/0003-4975(91)91373-4.

3. Iannettoni MD, Vlessis AA, Whyte RI, Orringer MB Functional Outcome after Surgical Treatment of Esophageal Perforation. The Annals of Thoracic Surgery. 1997;64(6):1606-1610.

4. Wesdorp IC, Bartelsman JF, Huibregtse K, den Hartog Jager FC, Tytgat GN. Treatment of instrumental oesophageal perforation. Gut. 1984;25(4):398-404.

5. Wu JT, Mattox KL, Wall MJ. Esophageal Perforations: New Perspectives and Treatment Paradigms. The Journal of Trauma: Injury, Infection, and Critical Care. 2007;63(5):11731184. doi: 10.1097/TA.0b013e31805c0dd4.

6. Abbas G, Schuchert MJ, Pettiford BL, Pennathur A Landreneau J, Landreneau J, Luketich JD, Landreneau RJ. Contemporaneous management of esophageal perforation. Surgery. 2009;146(4):749-755. doi: 10.1016/j. surg.2009.06.058.

7. Vaidya S, Prabhudessai S, Jhawar N, Patankar RV. Boerhaave's syndrome: Thoracolaparoscopic approach. Journal of Minimal Access Surgery. 2010;6(3):76-79. doi: 10.4103/0972-9941.68585.

8. Chikinev JuV, Drobjazgin EA, Poljakevich AS, Peshkova IV. Diagnostika i lechenie sindroma Burhave [Diagnostics and treatment of boerhaave's syndrome]. Vestnik hirurgii im. I.I. Grekova [Grekov's Bulletin of Surgery]. 2015;174(4):73-76. (Russian).
9. Kimura T, Takemoto T, Fujiwara Y, Yane K, Shiono H. Esophageal perforation caused by a fish bone treated with surgically indwelling drainage and fibrin glue injection for fistula formation. Ann Thorac Cardiovasc Surg. 2013;19(4):289-292. doi: 10.5761/atcs.cr.12.01906.

10. Chen Y-H, Li S-H, Chiu Y-C, Lu H-I, Huang C-H, Rau K-M, Liu C-T. Comparative study of esophageal stent and feeding gastrostomy/jejunostomy for tracheoesophageal fistula caused by esophageal squamous cell carcinoma. PLoS ONE. 2012;7(8):e42766. doi: 10.1371/journal. pone.0042766.

11. Graeber GM, Niezgoda JA, Albus RA, Burton NA, Collins GJ, Lough FC, Zajtchuk R. A comparison of patients with endoscopic esophageal perforations and patients with Boerhaave's syndrome. Chest. 1987;92(6):995-998. doi: 10.1378/chest.92.6.995.

12. Brinster CJ, Singhal S, Lee L, Marshall MB, Kaiser LR, Kucharczuk JC. Evolving options in the management of esophageal perforation. Ann Thorac Surg. 2004;77(4):14751483. doi: 10.1016/j.athoracsur.2003.08.037.

13. Young CA, Menias CO, Bhalla S, Prasad SR. CT features of esophageal emergencies. Radiographics. 2008;28(6):15411553. doi: 10.1148/rg.286085520.

14. Gupta A, Rizvi I, Srivastava VK, Debnath J, Kapur BN, Khanna VN, Aziz A. Spontaneous pneumomediastinum. Medical Journal Armed Forces India. 2015;71(Suppl 1):S85-S88. doi: 10.1016/j.mjafi.2013.03.008.

15. Ben-David K, Lopes J, Hochwald S, Draganov P, Forsmark C, Collins D, Chauhan S, Wagh MS, Carreras J, Vogel S, Sarosi G. Minimally invasive treatment of esophageal perforation using a multidisciplinary treatment algorithm: a 
case series. Endoscopy. 2011;43(2):160-162. doi: 10.1055/ $\mathrm{s}-0030-1256094$

16. Wahed S, Dent B, Jones R, Griffin SM. Spectrum of oesophageal perforations and their influence on management Br J Surg. 2014;101(1):e156-162. doi: 10.1002/bjs.9338.

17. Griffin SM, Lamb PJ, Shenfine J, Richardson DL, Karat D, Hayes N. Spontaneous rupture of the oesophagus. $\mathrm{Br} J$ Surg. 2008:95(9):1115-1120. doi: 10.1002/bjs.6294.

18. Santos GH, Frater RW. Transesophageal irrigation for the treatment of mediastinitis produced by esophageal rupture. J Thorac Cardiovasc Surg. 1986;91(1):57-62.

19. Hinojar AG, Castejón MAR, Hinojar AA. Conservative management of a case of cervical esophagus perforation with mediastinal abscess and bilateral pleural effusion. Auris Nasus Larynx. 2002;29(2):199-201.

20. Loske G. Endoscopic negative pressure therapy of the upper gastrointestinal tract. Chirurg. 2019;90(Suppl 1):1-6 doi: 10.1007/s00104-018-0727-x.

21. Mennigen R, Senninger N, Laukoetter MG. Novel treatment options for perforations of the upper gastrointestinal tract: Endoscopic vacuum therapy and over-the-scope clips. World J Gastroenterol. 2014;20(24):7767-7776. doi: 10.3748/wjg.v20.i24.7767.

22. Biancari F, D'Andrea V, Paone R, Di Marco C, Savino G, Koivukangas V, Saarnio J, Lucenteforte E. Current treatment and outcome of esophageal perforations in adults: systematic review and meta-analysis of 75 studies. World J Surg. 2013;37(5):1051-1059. doi: 10.1007/s00268-0131951-7.

23. Persson S, Elbe $P$, Rouvelas I, Lindblad M, Kumagai K, Lundell L, Nilsson M, Tsai JA. Predictors for failure of stent treatment for benign esophageal perforations - a single center 10-year experience. World J Gastroenterol. 2014;20(30):10613-10619. doi: 10.3748/wjg.v20.i30.10613

24. Dasari BVM Neely D, Kennedy A Spence G, Rice P Mackle $\mathrm{E}$, Epanomeritakis $\mathrm{E}$. The role of esophageal stents in the management of esophageal anastomotic leaks and benign esophageal perforations. Ann. Surg. 2014;259(5):852-860. doi: $10.1097 /$ SLA.0000000000000564

25. Koivukangas $V$, Biancari $F$, Meriläinen $S$, Ala-Kokko $T$, Saarnio J. Esophageal stenting for spontaneous esophageal perforation. J Trauma Acute Care Surg. 2012;73(4):1011 1013. doi: 10.1097/TA.0b013e318265d176.

26. Freeman RK, Ascioti AJ, Giannini T, Mahidhara RJ. Analysis of unsuccessful esophageal stent placements for esophageal perforation, fistula, or anastomotic leak. Ann Thorac Surg. 2012;94(3):959-964. doi: 10.1016/j.athoracsur.2012.05.047.

27. Sharma P, Kozarek R. Role of esophageal stents in benign and malignant disease. Am J Gastroenterol. 2010;105(2):258-273. doi: 10.1038/ajg.2009.684.

28. Babor R, Talbot M, Tyndal A. Treatment of upper gastrointestinal leaks with a removable, covered, self-expanding metallic stent. Surg Laparosc Endosc Percutan Tech. 2009;19(1):e1-4. doi: 10.1097/SLE.0b013e318196c706.

29. Muir AD, White J, McGuigan JA, McManus KG, Graham AN. Treatment and outcomes of oesophageal perforation in a tertiary referral centre. Eur J Cardiothorac Surg 2003;23(5):799-804. doi: 10.1016/s1010-7940(03)00050-2.

30. Hasimoto CN, Cataneo C, Eldib R, Thomazi R, Pereira RS de C, Minossi JG, Cataneo AJM. Efficacy of surgical versus conservative treatment in esophageal perforation: a systematic review of case series studies. Acta Cir Bras. 2013;28(4):266-271. doi: 10.1590/s010286502013000400006

31. Vogel SB, Rout WR, Martin TD, Abbitt PL. Esophageal Perforation in Adults: Aggressive, Conservative Treatment Lowers Morbidity and Mortality. Annals of Surgery. 2005;241(6):1016-1023. doi: 10.1097/01. sla.0000164183.91898.74

32. Kaman L, Iqbal J, Kundil B, Kochhar R. Management of Esophageal Perforation in Adults. Gastroenterology Research. 2010;3(6):235-244. doi: 10.4021/gr263w.
33. Collins C, Arumugasamy M, Larkin J, Martin S, O'Sullivan GC. Thoracoscopic repair of instrumental perforation of the oesophagus: first report. Ir J Med Sci. 2002;171(2):68-70. doi: 10.1007/BF03168953.

34. Jougon J, Mc Bride T, Delcambre F, Minniti A, Velly J-F. Primary esophageal repair for Boerhaave's syndrome whatever the free interval between perforation and treatment. Eur J Cardiothorac Surg. 2004;25(4):475-479. doi: 10.1016/j.ejcts.2003.12.029.

35. Tettey M, Edwin F, Aniteye E, Sereboe L, Tamatey $\mathrm{M}$, Entsua-Mensah $\mathrm{K}$, Kotei D, Frimpong-Boateng $\mathrm{K}$. Management of intrathoracic oesophageal perforation: analysis of 16 cases. Trop Doct. 2011;41(4):201-203. doi: 10.1258/td.2011.110120.

36. Kiss J. Surgical treatment of oesophageal perforation British Journal of Surgery. 2008;95(7):805-806.

37. Richardson JD. Management of esophageal perforations: the value of aggressive surgical treatment. $A m$ J Surg 2005;190(2):161-165. doi: 10.1016/j.amjsurg.2005.05.004.

38. Kollmar O, Lindemann W, Richter S, Steffen I, Pistorius G, Schilling MK. Boerhaave's syndrome: primary repair vs. esophageal resection--case reports and meta-analysis of the literature. J Gastrointest Surg. 2003;7(6):726-734. doi: 10.1016/s1091-255x(03)00110-0.

39. Lindenmann J, Matzi V, Neuboeck N, Anegg U, Maier A, Smolle J, Smolle-Juettner FM. Management of esophageal perforation in 120 consecutive patients: clinical impact of a structured treatment algorithm. J Gastrointest Surg. 2013;17(6):1036-1043. doi: 10.1007/s11605-012-2070-8.

40. Gouge TH, Depan HJ, Spencer FC. Experience with the Grillo pleural wrap procedure in 18 patients with perforation of the thoracic esophagus. Ann Surg. 1989;209(5):612-617. doi: $10.1097 / 00000658-198905000-00014$

41. Mahmodlou R, Abdirad I, Ghasemi-rad M. Aggressive Surgical Treatment in Late-Diagnosed Esophageal Perforation: A Report of 11 Cases. ISRN Surgery. 2011;2011:1-4. doi: 10.5402/2011/868356.

42. Abbott OA, Mansour KA, Logan WD, Hatcher CR, Symbas PN. Atraumatic so-called "spontaneous" rupture of the esophagus. A review of 47 personal cases with comments on a new method of surgical therapy. J Thorac Cardiovasc Surg. 1970;59(1):67-83.

43. Port JL, Kent MS, Korst RJ, Bacchetta M, Altorki NK Thoracic esophageal perforations: a decade of experience. The Annals of Thoracic Surgery. 2003;75(4):1071-1074. doi: 10.1016/s0003-4975(02)04650-7.

44. Linden PA, Bueno R, Mentzer SJ, Zellos L, Lebenthal A Colson YL, Sugarbaker DJ, Jaklitsch MT. Modified T-tube repair of delayed esophageal perforation results in a low mortality rate similar to that seen with acute perforations. Ann Thorac Surg. 2007;83(3):1129-1133. doi: 10.1016/j. athoracsur.2006.11.012.

45. Okonta KE, Kesieme EB. Is oesophagectomy or conservative treatment for delayed benign oesophageal perforation the better option? Interact Cardiovasc Thorac Surg. 2012;15(3):509-511. doi: 10.1093/icvts/ivs190.

46. Arciaga PL, Windokun A, Wood W, Frost EAM. Anesthetic management of iatrogenic esophageal perforation--case report and literature review. Middle East J Anaesthesiol. 2007:19(1):231-242.

47. Cho JS, Kim YD, Kim JW, I HS, Kim MS. Thoracoscopic primary esophageal repair in patients with Boerhaave's syndrome. Ann Thorac Surg. 2011;91(5):1552-1555. doi: 10.1016/j.athoracsur.2011.01.082.

48. Landen S, Nakadi El. Minimally invasive approach to Boerhaave's syndrome: a pilot study of three cases. Surg Endosc. 2002;16(9):1354-1357. doi: 10.1007/s00464-0019185-4.

49. Nguyen NT, Follette DM, Roberts PF, Goodnight JE. Thoracoscopic management of postoperative esophageal leak. J Thorac Cardiovasc Surg. 2001;121(2):391-392. doi: 10.1067/mtc.2001.110485.

50. Scott HJ, Rosin RD. Thoracoscopic repair of a transmural rupture of the oesophagus (Boerhaave's syndrome). J $R$ Soc Med. 1995;88(7):414P-415P. 
Конфликт интересов. Авторы заявляют об отсутствии конфликта интересов.

Финансирование. Исследование проведено без спонсорской поддержки.

Соответствие принципам этики. Исследование одобрено локальным этическим комитетом.

Сведения об авторах:

Игнатюк Александр Николаевич, УЗ «Брестская областная клиническая больница»; e-mail: alexihnatsiuk@gmail.com ORCID: 0000-0002-8925-380X

Карпицкий Александр Сергеевич, д-р мед. наук, проф.; УЗ «Брестская областная клиническая больница»; ORCID 0000-0001-8205-8959

Поступила: 03.01.2021

Принята к печати: 18.03.2021
Conflict of interest. The authors declare no conflict of interest.

Financing. The study was performed without external funding.

Conformity with the principles of ethics. The study was approved by the local ethics committee.

Information about authors:

Karpitsky Alexander Sergeevich; Health care institution «Brest Regional Clinical Hospital»; ORCID: 0000-0001-8205-8959

Ignatyuk Aleksandr Nikolaevich; Health care institution «Brest Regional Clinical Hospital»;

e-mail: alexihnatsiuk@gmail.com ORCID: 0000-0002-8925-380X

Received: 03.01.2021

Accepted: 18.03.2021
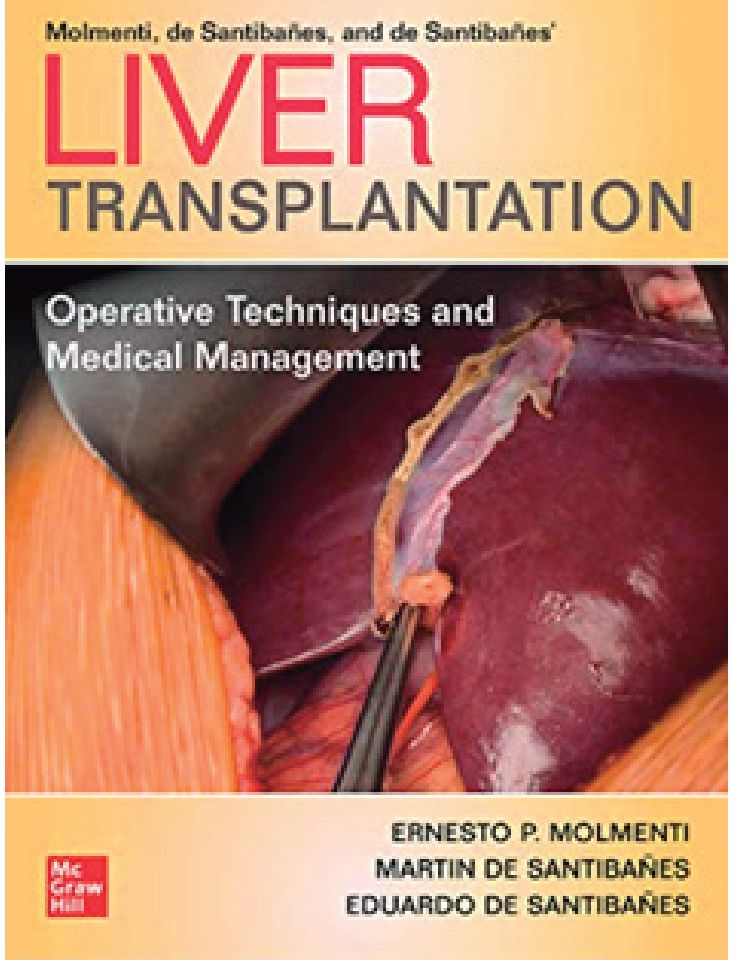

Molmenti, E. P. Liver Transplantation: Operative Techniques and Medical Management / E. P. Molmenti, M. de Santibañes, E. de Santibañes. - 1st ed. - New York : McGraw-Hill Education, 2021. - 752 p. - ISBN 978-1260462517.

A concise, illustrated guide to the operative techniques and medical management of liver transplantation Filled with more than 1,000 images, illustrations, and bulleted text, this invaluable book provides a highly visual approach to liver transplantations for a diverse audience of healthcare professionals who treat patients with acute and chronic liver failure that requires full or partial transplantation. With coverage of all topics in the field, this reader-friendly and practical book serves as an ideal reference for residents, fellows, and senior physicians. It is easy to read, simple, and practical. 